\title{
A Comparison of Salivary Steroid Levels during Diagnostic Tests for Adrenal Insufficiency
}

Michaela Dušková', Kateřina Šimůnková ${ }^{2}$, Jana Vítků', Lucie Sosvorová', Hana Jandíková', Hana Pospíšilová', Monika Šrámková', Mikuláš Kosák ${ }^{2}$, Michal Kršek², Václav Hána², Magdaléna Žánová ${ }^{3}$, Drahomíra Springer ${ }^{3}$, Luboslav Stárka ${ }^{1}$

${ }^{1}$ Institute of Endocrinology, Prague, Czech Republic;

${ }^{2} 3^{\text {rd }}$ Department of Medicine - Department of Endocrinology and Metabolism, First Faculty of Medicine, Charles University in Prague and General University Hospital in Prague, Prague, Czech Republic;

${ }^{3}$ Institute of Medical Biochemistry and Laboratory Diagnostics, First Faculty of Medicine, Charles University in Prague and General University Hospital in Prague, Prague, Czech Republic

Received December 2, 2015; Accepted February 24, 2016.

Key words: Saliva - Cortisol - Cortisone - Pregnenolone Dehydroepiandrosterone - Synacthen test - Insulin tolerance test

Abstract: Numerous diagnostic tests are used to evaluate the hypothalamicpituitary-adrenal axis (HPA axis). The gold standard is still considered the insulin tolerance test (ITT), but this test has many limitations. Current guidelines therefore recommend the Synacthen test first when an HPA axis insufficiency is suspected. However, the dose of Synacthen that is diagnostically most accurate and sensitive is still a matter of debate. We investigated 15 healthy men with mean/median age 27.4/26 (SD \pm 4.8 ) years, and mean/median BMI (body mass index) 25.38/24.82 $(S D \pm 3.2) \mathrm{kg} / \mathrm{m}^{2}$. All subjects underwent 4 dynamic tests of the HPA axis, specifically $1 \mu \mathrm{g}, 10 \mu \mathrm{g}$, and $250 \mu \mathrm{g}$ Synacthen (ACTH) tests and an ITT. Salivary cortisol, cortisone, pregnenolone, and DHEA (dehydroepiandrosterone) were analysed using liquid chromatography-tandem mass spectrometry. During the ITT maximum salivary cortisol levels over $12.5 \mathrm{nmol} / \mathrm{l}$ were found at

This study was supported by grant NT 11277-6 IGA of the Ministry of Health of the Czech Republic.

Mailing Address: Michaela Dušková, MD., PhD., Institute of Endocrinology, Národní 8, 11694 Prague 1, Czech Republic; Phone: +420 224905 412; e-mail:mduskova@endo.cz 
60 minutes. Maximum cortisol levels in all of the Synacthen tests were higher than this; however, demonstrating that sufficient stimulation of the adrenal glands was achieved. Cortisone reacted similarly as cortisol, i.e. we did not find any change in the ratio of cortisol to cortisone. Pregnenolone and DHEA were higher during the ITT, and their peaks preceded the cortisol peak. There was no increase of pregnenolone or DHEA in any of the Synacthen tests. We demonstrate that the $10 \mu \mathrm{g}$ Synacthen dose is sufficient stimulus for testing the HPA axis and is also a safe and cost-effective alternative. This dose also largely eliminates both false negative and false positive results.

\section{Introduction}

Diagnostic tests of the hypothalamic-pituitary-adrenal axis (HPA axis) are the subject of debate and still counter many difficulties. The gold standard for evaluating the HPA axis is considered to be the insulin tolerance test (ITT), but this test has several limitations and is not used in many countries. Another common test is the Synacthen (or ACTH) test, but various doses of the ACTH have been proposed (most commonly $1 \mu \mathrm{g}$ or $250 \mu \mathrm{g}$ ) and there is no consensus which dose is diagnostically most accurate and sensitive. The other limitation of all tests is in evaluating total cortisol levels in serum, since only free cortisol is biologically active and critical for a tissue response.

Total cortisol includes both free cortisol and cortisol bound to albumin, corticosteroid-binding globulin (CBG) and other plasma proteins. In the non-stimulated state about $90-95 \%$ of cortisol is bound to CBG, 5-10\% to albumin with low affinity, and 5-8\% exists in the free state (Torpy and Ho, 2007). Increasing and decreasing CBG concentrations in serum lead to changes in total serum cortisol levels. The free cortisol fraction remains unchanged, however, under the feedback control of the HPA axis. Despite this, changes in the concentrations of CBG and albumin have important consequences for interpretations results of clinical studies as well as for diagnosing HPA deficiencies. Changes in binding proteins may lead in miss-interpretation of results in conditions as renal insufficiency, critical illnesses, pregnancy and when using oral contraceptives (Ho et al., 2006; Qureshi et al., 2007).

For these reasons, when evaluating the HPA axis, measuring free cortisol would be preferable, since it avoids the influence of CBG (Mishra et al., 2007). Measuring of free cortisol levels in serum would have advantages because this reflects the biologically active cortisol fraction and acute changes in cortisol concentrations in the serum (Christ-Crain et al., 2007). Free cortisol fraction can be calculated, or measured in the laboratory. However, laboratory measurements are difficult and costly, and therefore are generally not performed for routine diagnostics (Vining et al., 1983; Klose et al., 2007). Other options are to use a calculated free cortisol index or calculate free cortisol according to the so-called Coolens' equation. The results, however, are often not satisfactory, since calculated values 
do not reflect free cortisol levels during changes in CBG and during low albumin levels throughout a dynamic test (Christ-Crain et al., 2007; Klose et al., 2007). For this reason, alternatives are being explored, the most promising of which seems to be measuring salivary cortisol levels (Šimůnková et al., 2007, 2008; Deutschbein et al., 2009b; Perogamvros et al., 2010). A measurement of salivary cortisol shows the ultrafilterable fraction, which reflects circulating levels of the free cortisol. Some studies have recommended measuring only basal cortisol concentrations for diagnostic purposes, and while basal serum cortisol is specific for $23 \%$ of patients with adrenal insufficiency, salivary cortisol is specific for $27 \%$ of such patients (Kazlauskaite et al., 2008; Deutschbein et al., 2009a). One of main problems is the wide variability in cortisol levels in both serum and saliva in the morning. For this reason, when an HPA axis deficiency is suspected, some authors recommend a Synacthen test rather than measuring basal cortisol concentrations (Artl, 2009).

Insufficiency of the HPA axis manifesting as adrenal insufficiency, is associated with higher morbidity and mortality. When this insufficiency is not diagnosed in time, it quickly leads to serious difficulties and sometimes even death of the patient. As shown in many studies, improperly indicated replacement therapies also carry significant metabolic risks. The ability to measure cortisol and other steroids in saliva as part of a diagnostic test of the HPA axis could make the diagnostic process easier and quicker for patients with changes in the concentration of protein-binding cortisol.

The aim of our study, therefore, was to evaluate salivary cortisol levels as well as those of cortisone, pregnenolone and dehydroepiandrosterone (DHEA) using liquid chromatography coupled with tandem mass spectrometry (LC-MS/MS) and chemiluminescent immunoassay (CLIA) Centaur XP Siemens in healthy volunteers, using both, the standard ITT test for diagnosing adrenal insufficiency and for comparing results with those from Synacthen tests using various concentrations of Synacthen (1 $\mathrm{gg}, 10 \mu \mathrm{g}$, or $250 \mu \mathrm{g})$.

\section{Methods}

Our study group consisted of 15 healthy men, with mean/median age 27.4/26 (SD \pm 4.8 ) years, and mean/median BMl (body mass index) 25.38/24.82 (SD \pm 3.2$) \mathrm{kg} / \mathrm{m}^{2}$. The men used no medications and had no history of using corticosteroids. All signed informed consent before starting the study, which was approved by the Ethical Commission of the Institute of Endocrinology. We performed four functional tests that are commonly used to diagnose adrenal insufficiency. The minimum time between tests was one week. The following tests were used: the $1 \mu \mathrm{g}$ "low dose" Synacthen test (LDST), the $10 \mu \mathrm{g}$ "medium dose" Synacthen test (MDST), the $250 \mu \mathrm{g}$ "high dose" Synacthen test (HDST), and the insulin tolerance test (ITT). All tests were performed after an overnight fast, and were started in the morning between 7 and 9 a.m. Each dose of Synacthen and insulin were given 
through a cannula inserted into the cubital vein, starting 15 minutes after cannula insertion.

Saliva was sampled at regular intervals during each test for measurements of steroid hormone levels. Saliva samples were collected in Sarstedt Salivette saliva examination tubes type 51.1534 , centrifuged at $1000 \mathrm{rpm}$ in the centrifuge, and frozen at $-20^{\circ} \mathrm{C}$.

The details of individual tests were as follows

LDST: The contents of 1 ampule $250 \mu \mathrm{g} / 1 \mathrm{ml}$ Synacthen (tetracosactide $250 \mu \mathrm{g}$, Novartis Pharma GmbH, Nuernberg, Germany) was added to $249 \mathrm{ml}$ physiological solution. This dilution was prepared at the day of the test. At the beginning of the test, $1 \mathrm{ml}$ of diluted solution was given intravenously, and saliva samples taken at 0 , $20,30,40$, and 60 minutes.

MDST: The contents of 1 ampule $250 \mu g / 1 \mathrm{ml}$ Synacthen (tetracosactide $250 \mu \mathrm{g}$, Novartis Pharma GmbH, Nuernberg, Germany) was added to $249 \mathrm{ml}$ physiological solution. This dilution was prepared in the day of the test. At the beginning of the test, $10 \mathrm{ml}$ of diluted solution were given intravenously, and saliva samples taken at $0,30,60$, and 90 minutes.

HDST: At the beginning of the test, the contents of 1 ampule $250 \mu \mathrm{g} / 1 \mathrm{ml}$ Synacthen (tetracosactide $250 \mu \mathrm{g}$, Novartis Pharma GmbH, Nuernberg, Germany) was given intravenously, and saliva samples taken at $0,30,60$, and 90 minutes.

ITT: At the beginning of the test $0.1 \mathrm{IU}$ per $1 \mathrm{~kg}$ Actrapid insulin was given intravenously. During the test blood glucose was regularly checked with a glucometer (Accu-Chek Perform), and blood pressure and pulse rate were measured every five minutes during the first hour and every ten minutes thereafter. There was a decrease in blood glucose below $2.2 \mathrm{mmol} / \mathrm{l}$ in all tests, and all patients had a spontaneous blood glucose response during the first hour followed by normalization. Saliva samples were taken at $0,20,30,40,60,90$, and 120 minutes.

Analyses of steroid levels

Cortisol, cortisone, pregnenolone, and dehydroepiandrosterone (DHEA) were measured using liquid chromatography coupled with tandem mass spectrometry (LC-MS/MS) (Sosvorova et al., 2015). In addition, cortisol was also measured using the chemiluminescent immunoassay (CLIA) Centaur XP Siemens (CLIA) method.

\section{Statistical analysis}

Data were transformed by Box-Cox transformation before further processing due to non-Gaussian distribution and non-constant variance (heteroscedasticity) in all variables. Repeated-measures analysis of variance (ANOVA) was used for monitoring levels of steroids during tests. Comparison of method for determination of cortisol in saliva was performed by single regression analysis. CLIA method was selected as a reference method because it is common method 
for clinical practice. The statistical software Statgraphics Centurion XVI from Statpoint Inc. (Warrenton, VA, USA) was used for data transformations, ANOVA testing.

\section{Results}

The results of salivary steroid levels measured during each test (maximum and minimum values for cortisol and cortisone) are given in Table 1.

\section{Cortisol (Figure 1)}

During the ITT test salivary cortisol levels reached a maximum of $12.5 \mathrm{nmol} / \mathrm{l}$ at $60 \mathrm{~min}$. This level was exceeded during all Synacthen test doses, demonstrating sufficient stimulation during all Synacthen tests. During the LDST there was a maximum increase in salivary cortisol at 30 minutes to over $15.9 \mathrm{nmol} / \mathrm{l}$, followed by a decrease. During the MDST there was a maximum increase at 60 minutes to over $21.1 \mathrm{nmol} / \mathrm{l}$, then a plateau until 90 minutes. During the HDST salivary cortisol continuously increased during the 90 minutes of the test to over $24.5 \mathrm{nmol} / \mathrm{l}$, and maximum levels likely occurred after the last sampling time.

Table 1 - Minimum and maximum levels of salivary cortisol and salivary cortisone from individual tests and times

\begin{tabular}{lccc}
\hline & Time $(\mathrm{min})$ & Salivary cortisol $(\mathrm{nmol} / \mathrm{l})$ & Salivary cortisone $(\mathrm{nmol} / \mathrm{l})$ \\
\hline \multirow{4}{*}{ LDST } & 0 & $2.3-19.4$ & $15.7-65.0$ \\
& 20 & $8.8-24.2$ & $34.1-73.3$ \\
& 30 & $15.9-41.7$ & $38.2-93.5$ \\
& 40 & $17.3-36.2$ & $39.4-79.7$ \\
MDST & 60 & $6.3-21.1$ & $29.2-70.9$ \\
& 0 & $1.8-19.6$ & $15.5-61.9$ \\
& 30 & $16.6-46.7$ & $33.9-104.7$ \\
& 60 & $21.1-66.3$ & $46.8-109.9$ \\
HDST & 90 & $22.2-56.6$ & $46.6-116.3$ \\
& 0 & $3.0-14.8$ & $22.1-56.4$ \\
& 30 & $11.5-35.1$ & $36.4-85.3$ \\
& 60 & $24.4-49.4$ & $39.0-126.6$ \\
& 90 & $24.5-74.8$ & $66.4-119.4$ \\
\hline \multirow{3}{*}{ ITT } & 0 & $2.9-18.4$ & $24.7-66.1$ \\
& 20 & $1.2-17.7$ & $16.2-72.7$ \\
& 30 & $2.0-19.3$ & $16.3-58.3$ \\
& 40 & $2.7-36.2$ & $17.0-59.1$ \\
& 60 & $12.5-32.6$ & $27.8-89.8$ \\
\hline
\end{tabular}

LDST - $1 \mu \mathrm{g}$ "low dose" Synacthen test; MDST - $10 \mu \mathrm{g}$ "medium dose" Synacthen test; HDST - 250 $\mu \mathrm{g}$ "high dose" Synacthen test; ITT - insulin tolerance test 

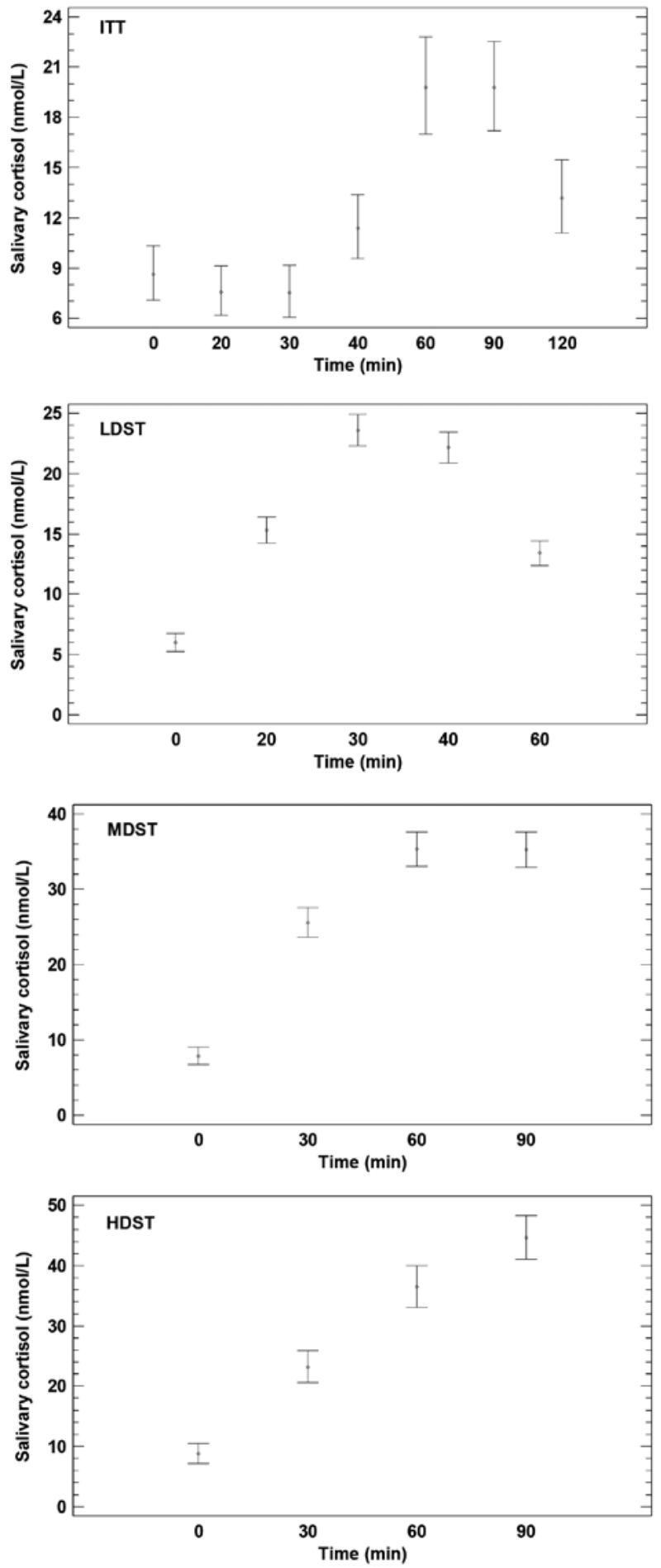

Figure 1 - Salivary cortisol levels over time in individual tests. 
24) Prague Medical Report / Vol. 117 (2016) No. 1, p. 18-33
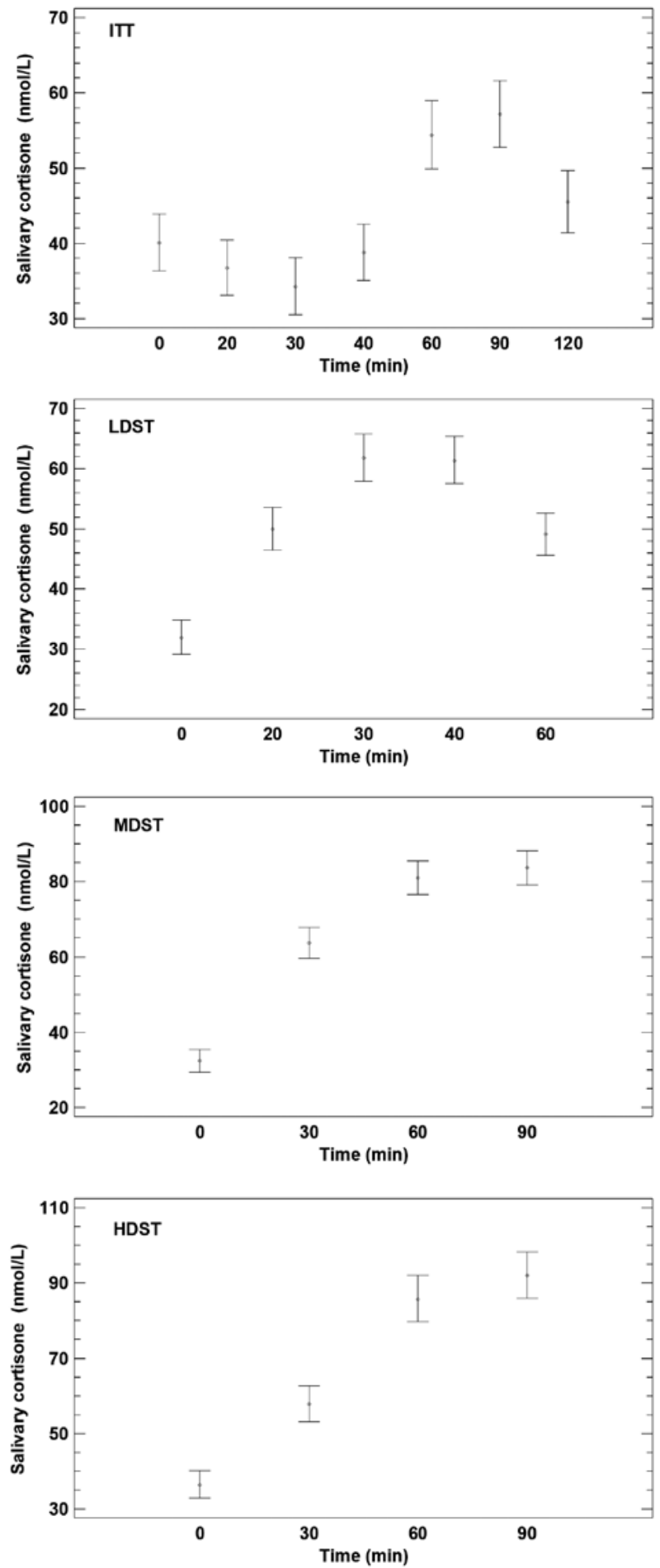

Figure 2 - Salivary cortisone levels over time in individual tests.

Dušková M. et al. 

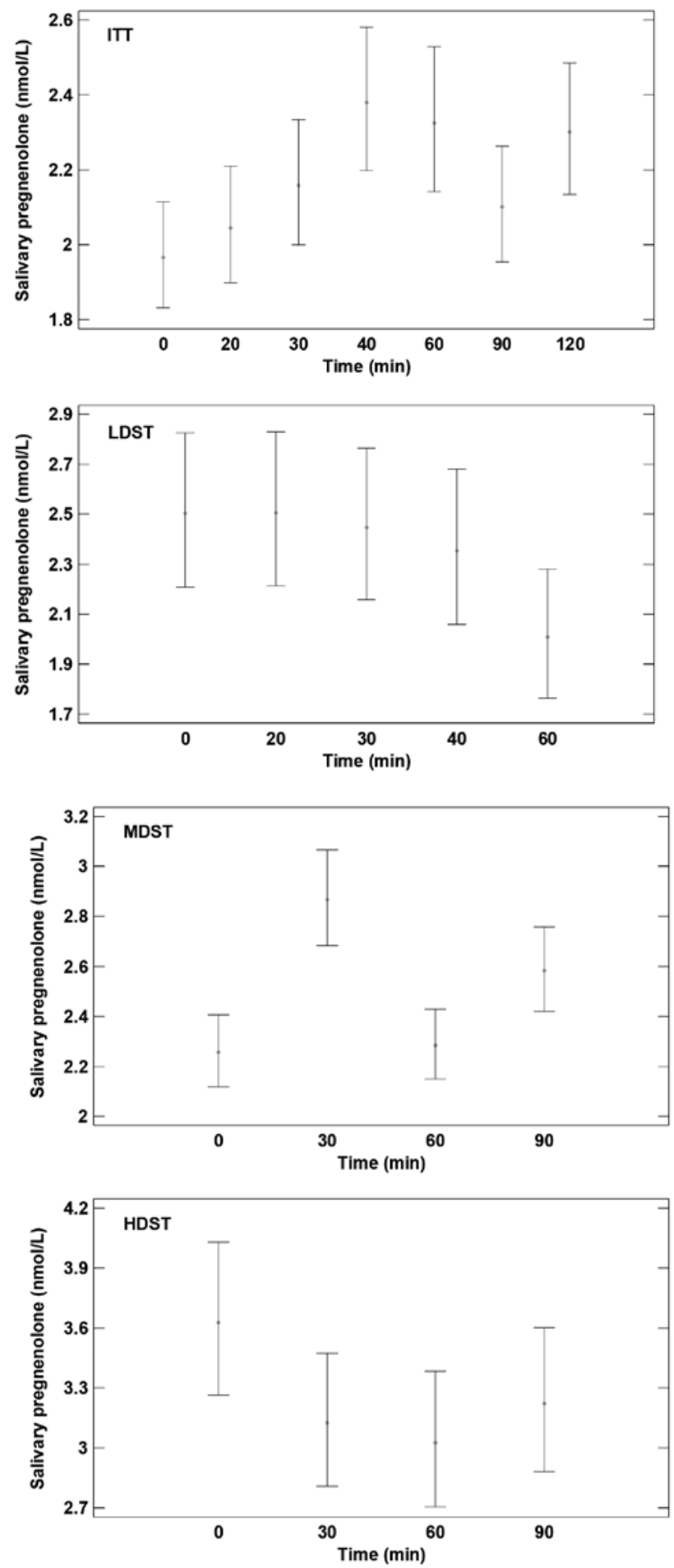

Figure 3 - Salivary pregnenolone levels over time in individual tests. 
26) Prague Medical Report / Vol. 117 (2016) No. 1, p. 18-33
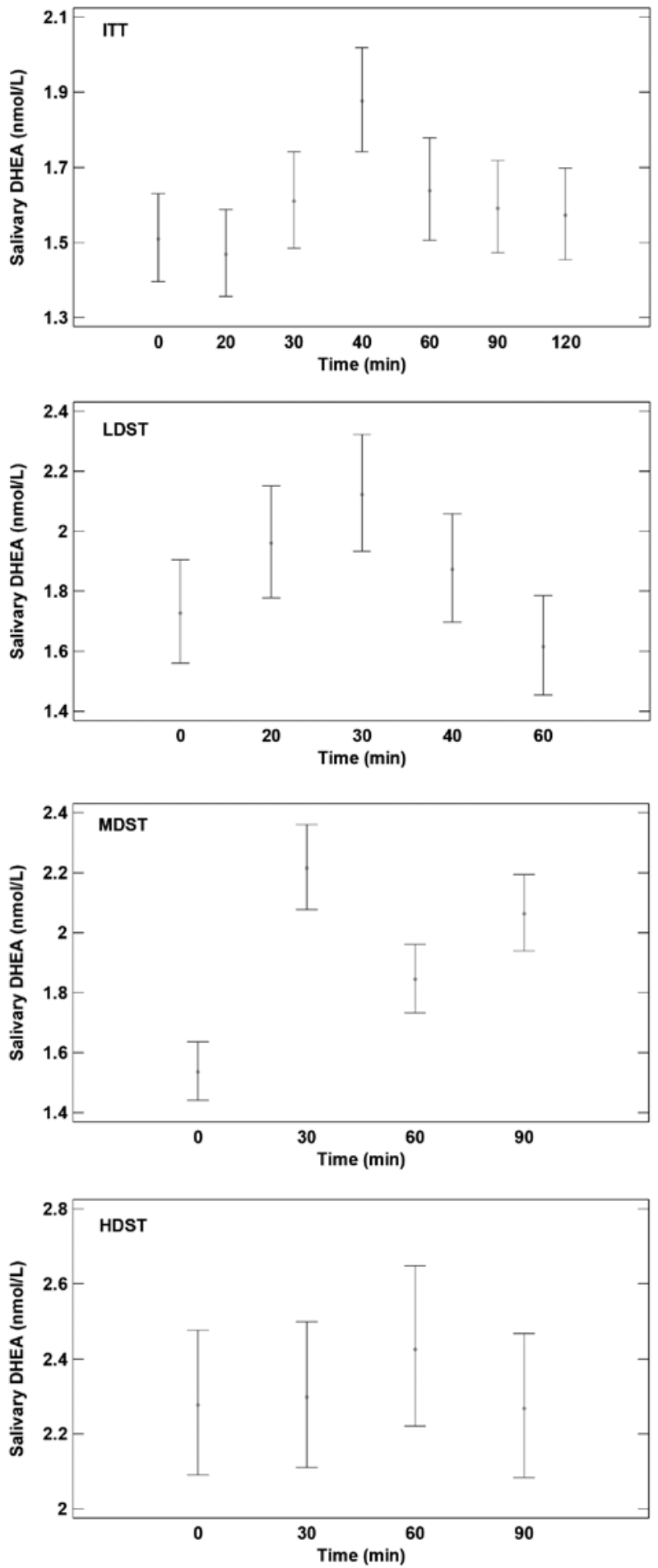

Figure 4 - Salivary DHEA (dehydroepiandrosterone) levels over time in individual tests.

Dušková M. et al. 


\section{Cortisone (Figure 2)}

During the ITT test salivary cortisone levels reached a maximum above $27.8 \mathrm{nmol} / \mathrm{I}$ between 60 and 90 minutes. This level was exceeded during all Synacthen test doses, again demonstrating sufficient stimulation during all tests. During the LDST there was a maximum increase in salivary cortisone by 30 minutes to over $38.2 \mathrm{nmol} / /$, followed by a decline. During the MDST there was a maximum increase in 60 minutes to over $21.2 \mathrm{nmol} / \mathrm{l}$, then a plateau until 90 minutes. During the HDST salivary cortisone continuously increased during the 90 minutes of the test to over $24.5 \mathrm{nmol} / \mathrm{l}$, and maximum levels likely occurred after the last sampling time.

\section{Pregnenolone (Figure 3)}

During the ITT test there was an increase in salivary pregnenolone in the first 40 minutes, a reaction of the adrenal glands to hypoglycemia. There was no increase in salivary pregnenolone during any of the Synacthen tests, indicating that salivary pregnenolone levels are not appropriate for evaluating the adrenal gland response during Synacthen tests.

\section{Dehydroepiandrosterone (Figure 4)}

During the ITT test there was an increase in salivary DHEA in the first 40 minutes, a reaction of the adrenal glands to hypoglycemia. Similarly as for salivary pregnenolone, there was no increase in salivary DHEA during any of the Synacthen tests, indicating that salivary DHEA levels are not appropriate for evaluating the adrenal gland response during Synacthen tests.

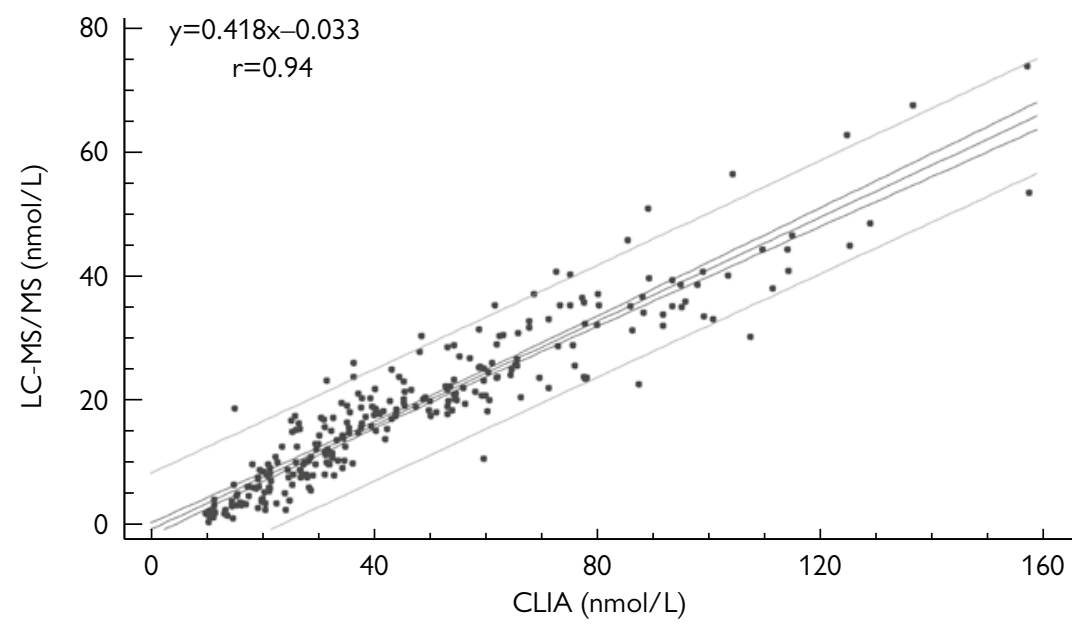

Figure 5 - Simple regression for cortisol levels. CLIA (chemiluminescent immunoassay) was used as the reference method $(x)$ and LC-MSIMS as the test method $(y)$. The regression line is surrounded by $95 \%$ confidence intervals (inner bounds). Outer bounds in the plot represent prediction intervals. 
Comparison of the LC-MSIMS method with a chemiluminescent immunoassay (CLIA) Centaur XP Siemens

We compared cortisol levels in 300 salivary samples measured by CLIA and LC-MS/MS method (Sosvorova et al., 2015). Using CLIA as a reference there was a strong correlation between the two methods $(r=0.94)$, with regression modelled by the equation $y=0.418 x-0.033$ (Figure 5). However, the slope of the regression line indicates that CLIA considerably overestimated cortisol levels.

\section{Discussion}

All tests demonstrated a sufficient stimulus for a steroid response in saliva, with the exception of pregnenolone and DHEA in the Synacthen test. In the goldstandard ITT test for adrenal insufficiency, the salivary cortisol maximum of above $12.5 \mathrm{nmol} / \mathrm{l}$ was reached by 60 minutes. This level was exceeded during all Synacthen dose tests, demonstrating sufficient stimulation of the adrenal glands in these tests. In our healthy study population, even the $1 \mu \mathrm{g}$ Synacthen dose induced a sufficient adrenal gland response, but the maximum cortisol concentration was reached earlier than during tests with higher Synacthen doses.

Maximum increase in serum cortisol levels during ITT tests have been reported to occur between 45 and 90 minutes (Borm et al., 2005). Maximum levels of salivary cortisol after stimulation have not been published, though recently a maximum stimulated salivary cortisol concentration of about $15 \mathrm{nmol} / \mathrm{l}$ after $250 \mu \mathrm{g}$ of ACTH has been demonstrated (Cornes et al., 2015), which is in line with the concentrations we found during the ITT test. Our unpublished data of serum cortisol showed also the lower cortisol response in ITT likely due to overstimulation of the adrenal gland during all of the ACTH tests. In addition, other reasons of the lower maximal stimulation level of salivary cortisol during ITT compared to all of the ACTH tests may lie in alteration of salivation induced by activation of autonomic nervous system during hypoglycemia (Ekström, 1989) and relatively worse performance of saliva collection due to affected consciousness during hypoglycemia. Our subjects response to insulin did not differ significantly in their severity of obtained hypoglycaemia nor or in BMI.

Maximum serum cortisol concentrations after various Synacthen doses show a dose-dependent response (Crowley et al., 1991). We also demonstrated this dosedependent response in salivary cortisol levels. For this reason, during lower-dose Synacthen tests, it is probably sufficient to sample saliva just during the first hour, though at more frequent intervals since the cortisol maximum may occur earlier (i.e. at $0,20,30,40$, and 60 minutes), as has been described for serum cortisol (Rasmuson et al., 1996; Patel and Clayton, 1999). Our results indicate that salivary cortisol during Synacthen tests behave similarly as serum cortisol levels.

The question remains, however, if stimulation in patients with altered HPA axis reactivity, for instance those with depressive symptomatology, might show false positive results (Sandström et al., 2011). This might burden patients with additional 
examinations or unnecessary treatment. However, stimulation with $250 \mu \mathrm{g}$ Synacthen is likely excessive. When testing serum cortisol levels this dose leads to repeated production of cortisol and maximum serum cortisol concentrations as long as 240 minutes after the Synacthen dosing (Crowley et al., 1991; Dicksten et al., 1997). In our study, maximum salivary cortisol levels had likely not yet been reached after 90 minutes.

As has already been described, such hyperstimulation can stimulate insufficient adrenal gland function in the subclinical phase of adrenal insufficiency and leads to false negative results, which present a high risk of underdiagnosing subclinical forms of adrenal insufficiency (Landon et al., 1984; Poršová et al., 1987; Dickstein et al., 1991). Full CBG saturation occurs at serum cortisol concentrations of 400-500 nmol//, after which the free serum cortisol fraction changes exponentially (Torpy and Ho, 2007). During stressful situations, increased cortisol can exceed the capacity of CBG and free cortisol can increase by up to $20 \%$, leading to marked changes in salivary cortisol concentrations (Torpy and Ho, 2007).

We suggest using a Synacthen dose of $10 \mu g$, despite the fact that this remains a supra-physiological stimulus. A maximum cortisol response to Synacthen is achieving by administration of 12-14 pmol/l ACTH and hence the dose of $0.5 \mu \mathrm{g}$ Synacthen is also supraphysiological (Oelkers, 1996). However, a $10 \mu \mathrm{g}$ Synacthen dose avoids some of the limitations that arise from a $1 \mu \mathrm{g}$ dose. It has been reported that a $1 \mu \mathrm{g}$ Synacthen dose can lead to an insufficient response in some healthy individuals (Laureti et al., 1998, 2000, 2002). Reasons behind this might be, for instance, an incorrectly mixed or prepared dose solution, the fact that Synacthen might adhere to the plastic cannula walls, or the cannula not being properly flushed so that the entire Synacthen dose reaches the circulation. There is no unanimous method for giving Synacthen - some prefer intramuscular injections, others intravenous (Wallace et al., 2009; Chatha et al., 2010). However, it is clear that $1 \mu \mathrm{g}$ Synacthen should only be given intravenously, since only $24 \%$ of intramuscularly injected Synacthen actually reaches the circulation (Dickstein, 1998). The possibility therefore exists when giving a $1 \mu \mathrm{g}$ Synacthen dose that the patient does not actually receive any of the test drugs (Dickstein et al., 1991; Murphy et al., 1998; Agha et al., 2006; Wallace et al., 2009; Chatha et al., 2010).

In our results, the cortisone response in saliva mirrored that of cortisol, but reached higher concentrations in all of the tests, likely due to the activity of $11 \beta$-hydroxysteroid dehydrogenase type 2 (HSD2) in the saliva that converts cortisol to cortisone. Perogamvros et al. (2010) found that during stimulation by $250 \mu \mathrm{g}$ Synacthen cortisone was stable, and recommended this as an alternative parameter to free cortisol when evaluating adrenal capacity. A recently published study has described a linear and bimodal correlation between cortisol and cortisone in saliva in basal levels and as well as after stimulation which agrees with the results we found in all our tests (Cornes et al., 2015). Altered 11ß-HSD2 activity by genetic defects or by medication and diet (derivatives of glycyrrhetinic 
acid) may lead to changes in salivary cortisol and cortisone that fact has to be taken into account and excluded (van Uum et al., 2002; Perogamvros et al., 2010).

The reaction of pregnenolone and DHEA during the tests is noteworthy. During the hypoglycemia test there were increases in both at 40 minutes, before the increase in cortisol. The ITT confirms adrenal function and the response can be seen in all steroids. This is in line with the assumption that ACTH is not a regulator of the adrenal biosynthesis of adrenal androgens, that is, mainly of that of DHEA. This applies also to pregnenolone as well. The tests with both high and low doses of Synacthen showed that there is a depletion of adrenal pregnenolone as a precursor of other hormones. The determining factor for the production of adrenal androgens has yet to be found, however.

The analyte concentrations presented in this paper were measured by LC-MS/MS. However, in routine laboratory settings immunochemical methods are most common. We compared salivary cortisol concentrations measured by both LC-MS/MS and CLIA, and found them to be highly correlated $(r=0.94)$, however CLIA overestimated absolute values. This is in agreement with our previous results, where we compared serum cortisol concentrations using LC-MS/MS and a radioimmunoassay (RIA) (Dušková et al., 2015). The study of Vieira et al. (2014) compared salivary cortisol measured by LC-MS/MS and an in-house RIA method and also found a high correlation $(r=0.82)$.

Results obtained when using an immunoassay also depend on the type of analyzer used.The study of Cornes et al. (2015) compared serum cortisol levels after the administration of $250 \mu \mathrm{g}$ of Synacthen using immunoassays measured on Roche and Abbot analyzers with measurements from LC-MS/MS. Results were highly correlated, but values from the Abbott analyzer were about $20 \%$ lower than those from the Roche analyzer. The overestimation of values by immunoassays and the differences between individual analyzers all need to be taken into account when evaluating results in clinical practice. Despite this, the availability and costeffectiveness of immunoassay methods remain the method of choice for routine clinical settings (Taylor et al., 2015).

\section{Conclusion}

Our results indicate that a $10 \mu \mathrm{g}$ Synacthen dose is a safe and cost-effective choice when testing patients suspected of adrenal insufficiency, which also largely eliminates the risk of false positive and false negative results. In addition, the $10 \mu \mathrm{g}$ dose can be more easily diluted in hospital pharmacies, allowing a precise dose to be given. In light of the expiration time of one month after dilution, this dose also allows the testing of as many patients as possible from one $250 \mu \mathrm{g}$ ampule of Synacthen, making this dosing cost-effective. This issue is even more critical considering the fact that Synacthen production was recently halted for a number of months.

When evaluating test results, the methods of analysis and associated reference ranges must always be taken into account. Cortisol levels measured by different 
methods may be highly correlated, but absolute values can vary by tens of percent. It is critical that each practice determine its own guidelines for a sufficient response to dynamic test of adrenal insufficiency.

\section{References}

Agha, A., Tomlinson, J.W., Clark, P. M., Holder, G., Stewart, P. M. (2006) The long-term predictive accuracy of the short synacthen (corticotrophin) stimulation test for assessment of the hypothalamic-pituitaryadrenal axis. J. Clin. Endocrinol. Metab. 91(1), 43-47.

Artl,W. (2009) The approach to the adult with newly diagnosed adrenal insufficiency. J. Clin. Endocrinol. Metab. 94, 1059-1067.

Borm, K., Slawik, M., Beuschlein, F., Seiler, L., Flohr, F., Berg, A., Koenig, A., Reincke, M. (2005) Low-dose glucose infusion after achieving critical hypoglycemia during insulin tolerance testing: Effects on time of hypoglycemia, neuroendocrine stress response and patient's discomfort in a pilot study. Eur. J. Endocrinol. 153(4), 521-526.

Chatha, K. K., Middle, J. G., Kilpatrick, E. S. (2010) National UK audit of the short synacthen test. Ann. Clin. Biochem. 47(Pt 2), 158-164.

Christ-Crain, M., Jutla, S., Widmer, I., Couppis, O., König, C., Pargger, H., Puder, J., Edwards, R., Müller, B., Grossman, A. B. (2007) Masurement of serum free cortisol shows discordant responsivity to stress and dynamic evaluation. J. Clin. Endocrinol. Metab. 92, 1729-1735.

Cornes, M. P., Ashby, H. L., Khalid, Y., Buch, H. N., Ford, C., Gama, R. (2015) Salivary cortisol and cortisone responses to tetracosactrin (synacthen). Ann. Clin. Biochem. 52(Pt 5), 606-610.

Crowley, S., Hindmarsh, P. C., Holownia, P., Honour, J.W., Brook, C. G. (1991) The use of low doses of ACTH in the investigation of adrenal function in man. J. Endocrinol. 130, 475-479.

Deutschbein, T., Unger, N., Mann, K., Petersenn, S. (2009a) Diagnosis of secondary adrenal insufficiency: Unstimulated early morning cortisol in saliva and serum in comparison with the insulin tolerance test. Horm. Metab. Res. 41(11), 834-839.

Deutschbein, T., Unger, N., Mann, K., Petersenn, S. (2009b) Diagnosis of secondary adrenal insufficiency in patients with hypothalamic-pituitary disease: Comparison between serum and salivary cortisol during the high-dose short synacthen test. Eur. J. Endocrinol. 160, 9-16.

Dickstein, G. (1998) The low dose (1 microgram) ACTH test - When and how to use it. Clin. Endocrinol. (Oxf.) 49(1), 135.

Dickstein, G., Shechner, C., Nicholson, W. E., Rosner, I., Shen-Orr, Z., Adawi, F., Lahav, M. (1991) Adrenocorticotropin stimulation test: Effects of basal cortisol level, time of day, and suggested new sensitive low dose test. J. Clin. Endocrinol. Metab. 72(4), 773-778.

Dickstein, G., Spigel, D., Arad, E., Shechner, C. (1997) One microgram is the lowest ACTH dose to cause a maximal cortisol response. There is no diurnal variation of cortisol response to submaximal $A C T H$ stimulation. Eur. J. Endocrinol. 137, 172-175.

Dušková, M., Sosvorová, L., Vítků, J., Jandíková, H., Rácz, B., Chlupáčová, T., De Cordeiro, J., Stárka, L. (2015) Changes in the concentrations of corticoid metabolites - The effect of stress, diet and analytical method. Prague Med. Rep. 116(4), 268-278.

Ekström, J. (1989) Autonomic control of salivary secretion. Proc. Finn. Dent. Soc. 85, 323-331.

Ho, J. T., Al-Musalhi, H., Chapman, M. J., Quach, T., Thomas, P. D., Bagley, C. J., Lewis, J. G., Torpy, D. J. (2006) Septic shock and sepsis: A comparison of total and free plasma cortisol levels. J. Clin. Endocrinol. Metab. 91, 105-114.

Kazlauskaite, R., Evans, A. T., Villabona, C.V., Abdu, T. A., Ambrosi, B., Atkinson, A. B., Choi, C. H., Clayton, R. N., Courtney, C. H., Gonc, E. N., Maghnie, M., Rose, S. R., Soule, S. G., Tordjman, K.; Consortium for 
Evaluation of Corticotropin Test in Hypothalamic-Pituitary Adrenal Insufficiency (2008) Corticotropin tests for hypothalamic-pituitary-adrenal insufficiency: a metaanalysis. J. Clin. Endocrinol. Metab. 93(11), 4245-4253.

Klose, M., Lange, M., Rasmussen, A. K., Skakkebæk, N. E., Hilsted, L., Haug, E., Andersen, M., Feldt-

Rasmussen, U. (2007) Factors influencing the adrenocorticotropin test: Role of contemporary cortisol assays, body composition, and oral contraceptive agents. J. Clin. Endocrinol. Metab. 92, 1326-1333.

Landon, J., Smith, D. C., Perry, L. A. (1984) The assay of salivary cortisol. In: Immunoassays of Steroids in Saliva; Proceedings of the Ninth Tenovus Workshop, Cardiff, UK, 1982. Read, G. F., Editor, pp. 300-307, Alpha Omega Publishing Ltd., Cardiff.

Laureti, S., Aubourg, P., Calcinaro, F., Rocchiccioli, F., Casucci, G., Angeletti, G., Bruneti, P., Falorni, A. (1998) Etiological diagnosis of primary adrenal insufficiency using an original flowchart of immune and biochemical markers. J. Clin. Endocrinol. Metab. 83, 3163-3168.

Laureti, S., Arvat, E., Candeloro, P., Di Vito, L., Ghigo, E., Santeusanio, F., Falorni, A. (2000) Low dose (1 microg) $\mathrm{ACTH}$ test in the evaluation of adrenal dysfunction in pre-clinical Addison's disease. Clin. Endocrinol. (Oxf.) 53(1), 107-115.

Laureti, S., Candeloro, P., Aglietti, M. C., Giordano, R., Arvat, E., Ghigo, E., Santeusanio, F., Falorni, A. (2002) Dehydroepiandrosterone, 17alpha-hydroxyprogesterone and aldosterone responses to the low-dose (1 micro g) ACTH test in subjects with preclinical adrenal autoimmunity. Clin. Endocrinol. (Oxf.) 57(5), 677-683.

Mishra, S. K., Gusta, N., Goswami, R. (2007) Plasma adrenocorticotropin (ACTH) values and cortisol response to 250 and $1 \mu \mathrm{g} \mathrm{ACTH}$ stimulation in patients with hyperthyroidism before and after carbimazole therapy: case-control comparative study. J. Clin. Endocrinol. Metab. 92, 1693-1696.

Murphy, H., Livesey, J., Espiner, E. A., Donald, R. A. (1998) The low dose ACTH test - a further test. Ann. Clin. Biochem. 47(Pt 2), 158-164.

Oelkers, W. (1996) Dose-response aspects in the clinical assessment of the hypothalamo-pituitary-adrenal axis, and the low-dose adrenocorticotropin test. Eur. J. Endocrinol. 135, 27-33.

Patel, L., Clayton, P. E. (1999) Clinical usefulness of the low dose ACTH test. J. Endocrinol. Invest. 2(5), 401-404.

Perogamvros, I., Keevin, B. G., Ray, D.W., Trainer, P. J. (2010) Salivary cortisone is potential biomarker for serum free cortisol. J. Clin. Endocrinol. Metab. 95, 4951-4958.

Poršová, I., Stárka, L., Blahoš, J., Putz, Z. (1987) Graduated intrasmuscular ACTH test with measurement of salivary cortisol. Vnitr. Lek. 33, 356-360. (in Czech)

Qureshi, A. C., Bahri, A., Breen, L. A., Barnes, S. C., Powrie, J. K., Thomas, S. M., Carroll, P.V. (2007) The influence of the route of oestrogen administration on serum levels of cortisol-binding globulin and total cortisol. Clin. Endocrinol. (Oxf.) 66, 632-635.

Rasmuson, S., Olsson, T., Hagg, E. (1996) A low dose ACTH test to assess the function of the hypothalamicpituitary-adrenal axis. Clin. Endocrinol. (Oxf.) 44(2), 151-156.

Sandström, A., Peterson, J., Sandström, E., Lundberg, M., Nystrom, I. L., Nyberg, L., Olsson, T. (2011) Cognitive deficits in relation to personality type and hypothalamic-pituitary-adrenal (HPA) axis dysfunction in women with stress-related exhaustion. Scand. J. Psychol. 52(1), 71-82.

Šimůnková, K., Hampl, R., Hill, M., Doucha, J., Stárka, L., Vondra, K. (2007) Salivary cortisol in low dose (1 microg) ACTH test in healthy women: comparison with serum cortisol. Physiol. Res. 56(4), 449-453.

Šimůnková, K., Stárka, L., Hill, M., Kř̌ž, L., Hampl, R., Vondra, K. (2008) Comparison of total and salivary cortisol in a low-dose ACTH (Synacthen) test: Influence of three-month oral contraceptives administration to healthy women. Physiol. Res. 57, S193-S199 (Suppl. 1). 
Sosvorova, L., Vitku, J., Chlupacova, T., Mohapl, M., Hampl, R. (2015) Determination of seven selected neuroand immunomodulatory steroids in human cerebrospinal fluid and plasma using LC-MS/MS. Steroids $\mathbf{9 8 ,}$ $1-8$.

Taylor, A. E., Keevil, B., Huhtaniemi, I. T. (2015) Mass spectrometry and immunoassay: How to measure steroid hormones today and tomorrow. Eur. J. Endocrinol. 173(2), D1-D12.

Torpy, J. T., Ho, D. J. (2007) Corticosteroid-binding globulin gene polymorphisms: Clinical implications and links to idiopathic chronic fatigue disorders. Clin. Endocrinol. (Oxf.) 67, 161-167.

van Uum, S. H., Walker, B. R., Hermus, A. R., Sweep, C. G., Smits, P., de Leeuw, P.W., Lenders, J.W. (2002) Effect of glycyrrhetinic acid on $11 \beta$-hydroxysteroid dehydrogenase activity in normotensive and hypertensive subjects. Clin. Sci. (Lond.) 102, 203-211.

Vieira, J. G., Nakamura, O. H., Carvalho, V. M. (2014) Determination of cortisol and cortisone in human saliva by a liquid chromatography-tandem mass spectrometry method. Arq. Bras. Endocrinol. Metabol. 58(8), 844-850.

Vining, R. F., McGinley, R. A., Maksvytis, J. J., Ho, K.Y. (1983) Salivary cortisol:A better measure of adrenal cortical function than serum cortisol. Ann. Clin. Biochem. 20(Pt 6), 329-335.

Wallace, I., Cunningham, S., Lindsay, J. (2009) The diagnosis and investigation of adrenal word of caution. J. Clin. Endocrinol. Metab. 83(2), 712-713. 